Revista de Política Económica para el Desarrollo Sostenible

Revista Electrónica Semestral, ISSN-2215-4167

Volumen 1, número 2

Enero - junio, 2016

http:// http://www.revistas.una.ac.crl

\title{
Organizaciones de pequeños productores, certificaciones y competitividad: experiencia reciente en Costa Rica
}

Rafael Diaz Porras, Antonio Delgado Ballesteros y William Páez Ramírez 


\title{
Organizaciones de pequeños productores, certificaciones y competitividad: experiencia reciente en Costa Rica
}

\author{
Organizations of small producers, certifications and competitiveness: recent experience in Costa \\ Rica
}

\section{RESUMEN}

\author{
Rafael Díaz Porras ${ }^{1}$ \\ Antonio Delgado Ballestero ${ }^{2}$ \\ William Páez Ramírez
}

\begin{abstract}
Las crecientes regulaciones privadas y públicas a los procesos productivos con requerimientos cada vez más fuertes para participar en los mercados han llevado a los pequeños y medianos productores a enfrentar numerosas encrucijadas cumpliendo dichas exigencias. Este artículo indaga si la aplicación de certificaciones ambientales ha significado un mejoramiento de la competitividad de los pequeños y medianos productores agrícolas. Para ello, se realiza un análisis del entorno de las certificaciones tanto internacional como en la institucionalidad en Costa Rica y se hace una aproximación a las realidades de las experiencias de las organizaciones de pequeños productores con base en los resultados de una encuesta realizada en el año 2015. El artículo concluye que la estrategia de certificarse ha permitido un mejor posicionamiento y, por consiguiente, estabilidad en la cadena de valor, aunque para las organizaciones y las instancias generadoras de políticas hay áreas donde mejorar, como por ejemplo los costos y cambios en los procesos y la resistencia cultural a este tipo de iniciativas.
\end{abstract}

Palabras Claves: certificaciones, productores agrícolas, organizaciones, competitividad.

\section{ABSTRACT}

Increasing private and public regulations to production processes with stringent requirements to participate in markets have led small and medium producers to face numerous crossroads to meet those requirements. This article investigates whether the application of environmental certifications has resulted in an improvement in the competitiveness of small and medium growers. The analysis focuses on the background of the certifications both at international and local levels, and approaches the real experiences of small producer organizations based on a survey conducted in 2015. This paper concludes that the strategy of obtaining certifications has improved grower organizations positioning and, consequently, their stability in the value chain. Nevertheless, organizations and entities generating policies face important areas for improvement, such as costs and changes in production processes and cultural resistance to this type of initiatives.

Keywords: certifications, small growers, organizations, competitiveness.

\footnotetext{
${ }^{1}$ Ph.D en Economía por la Universidad de Tilburg, Holanda. Catedrático del Centro Internacional de Política Económica para el Desarrollo Sostenible, Universidad Nacional (UNA)

2 Máster en Sistemas de Información por el Instituto Tecnológico de Costa Rica. Docente-investigador Centro Internacional de Política Económica para el Desarrollo Sostenible y Escuela de Sociología, Universidad Nacional (UNA).

${ }^{3}$ Máster en Política Económica por el Centro Internacional de Política Económica para el Desarrollo Sostenible. Director del Programa de Abastecimiento y Apoyo de la Universidad Nacional (UNA).
} 


\section{Introducción}

Actualmente es reconocido que el vínculo natural de la agricultura con los recursos naturales ha llevado a problemas de sostenibilidad. Los distintos balances de los impactos ambientales evidencian a la agricultura como una fuente importante del problema. Sin embargo, las soluciones asociadas a los sistemas de certificación que se han propuesto no garantizan la sostenibilidad integral de la agricultura. Por otra parte, se generan crecientes regulaciones privadas y públicas a los procesos productivos con requerimientos cada vez más fuertes para participar en los mercados. De esta forma, pequeños y medianos productores enfrentan numerosas encrucijadas cumpliendo dichas exigencias para acceder a los mercados, pero sin suficiente garantía de que estos remuneren su esfuerzo. Es necesario aclarar los alcances para los pequeños productores cuando desarrollan estrategias de certificación y de los cambios que se deben incorporar en los procesos productivos; además de las políticas que se requieren en el nivel nacional, que permitan el efectivo reconocimiento por parte de los mercados y las cadenas de comercialización a los agricultores.

La discusión se basa en los resultados obtenidos en el marco del proyecto "015512 Certificaciones ambientales: mejoramiento de la competitividad de los pequeños productores. Experiencias de Costa Rica 1990-2012", desarrollado en el Centro Internacional de Política Económica para el Desarrollo Sostenible (CINPE) de la Universidad Nacional de Costa Rica.

Las certificaciones ambientales aplicadas a los procesos productivos de agroexportación han concretado, por una parte, un impulso proveniente desde la demanda (Abarca y Sepúlveda, 2001, págs. 1-3), con el surgimiento de nichos de mercado en los cuales se reconoce un sobreprecio a los productos (Liu, 2010, pág. 139) que de alguna forma mejoran su perfil ambiental, pues se les asocia con la diferenciación del producto (Bingen y Busch, 2006, pág. 8). Asimismo, los requerimientos de dichas regulaciones se vinculan con la creciente preocupación ciudadana local por los efectos de la presión sobre los sistemas productivos y de sus impactos en el ambiente y en la salud ${ }^{4}$. La problemática del cambio climático agrega a esta situación una dimensión global, por implicar impactos transfronterizos.

Como resultado de las estrategias seguidas, se tiene una amplia gama de certificaciones que los productores pueden adoptar unilateralmente, si quisieran acceder a los nichos de mercado que se declaren social y ambientalmente responsables, tanto en el exterior como en el mercado local. Para los pequeños productores se presentan estos esquemas como oportunidades de acceso a nichos de mercado y valorización de sus recursos. Como esta estrategia se enfoca hacia nichos de mercado, surge la interrogante

\footnotetext{
${ }^{4}$ Auld reporta sobre la creciente percepción de los consumidores respecto a la producción de café en los esquemas de producción orgánica y comercio justo (Auld, 2011, pág. 69).
} 
de hasta dónde la apuesta que realizan los pequeños productores les significa una mayor estabilidad de su presencia en el mercado.

Por otra parte, en el tanto en que el mejoramiento del perfil ambiental de la producción agrícola da posibilidades de creación de valor agregado en la cadena, esto implica que los mencionados nichos de mercado son espacios promisorios, no solo para agentes alternativos a los tradicionales, sino que estos mismos participan directamente de estos nichos. El resultado es que los requerimientos de la gestión ambiental se agregan a los de calidad y, por lo tanto, presentan una tendencia en dos líneas: por una parte, que las empresas dominantes en los mercados accedan a los nichos ambientalmente amigables y, por otra, que la diferenciación tienda a ser una norma.

Lo anterior hace de la producción ambientalmente amigable un espacio para las empresas transnacionales, ya sea en lo productivo como en lo comercial, con el aprovechamiento de escalas de mayor producción, que lleva a la preocupación por la gobernanza de este proceso (ETC GROUP, 2012). El significado de estas tendencias para los pequeños productores agrícolas podría ser incierto. Esto lleva a pensar respecto a la presencia de contra tendencias, ya sea por la vía del desarrollo de escalas y altas productividades, restringiendo el espacio a la posibilidad de la pequeña producción, o manteniendo la presencia de la pequeña producción, aunque la gobernanza de las cadenas en que esta participa podría tender a niveles de mayor subordinación.

Las aristas que fundamentan esta preocupación central refieren a:

- Grado de fortaleza de las barreras de entrada: una estrategia de nicho implica desarrollo de barreras de entrada a la competencia que permitan mantener el posicionamiento alcanzado. Sin embargo, la proliferación de certificaciones y la reconocida confusión que genera en los mercados, particularmente en los consumidores, significa una fisura en esa estrategia para los productores agrícolas.

- El costo de las certificaciones y su permanencia en el tiempo: este es un esfuerzo demandante de sistematización, inversión y aprendizaje para las organizaciones productivas que lo realicen. En el caso de los pequeños productores, los procesos de certificación los obliga a abordarlo principalmente a partir de estrategias colectivas lideradas por sus organizaciones.

- Implementación de sistemas de certificación con menores requerimientos, focalizado en algunos aspectos, para efectos de lograr una mayor cobertura de productores y transmitir a los mercados una imagen verde. Esta podría ser una estrategia de acceso a mercados más amplios, lo cual reduce la diferenciación y se aproxima a la normalización. 
- Traducción de las certificaciones, como un ejercicio privado al ámbito de las regulaciones gubernamentales, que tiende a su normalización, convirtiéndolos en condiciones de acceso a mercados. Esto hace que se transforme su adopción por parte de los productores más en una estrategia de acceso al mercado y menos en diferenciación de productos.

Dado lo anterior, al indagar si la aplicación de certificaciones ambientales ha significado un mejoramiento de la competitividad de los pequeños y medianos productores agrícolas, surgen las siguientes preguntas:

1) ¿Qué tanto permanece en el tiempo la certificación ambiental por parte de los productores? ¿El esfuerzo y su costo han sido asimilados por los productores?

2) ¿Han significado las certificaciones acceso a nichos de mercado de mejor precio al producto y barreras de entradas sostenidas en el tiempo, tanto en los mercados internacionales como en los locales?

3) ¿Las certificaciones han permitido un mejor posicionamiento de las organizaciones de pequeños productores en la cadena de valor?

4) ¿La evolución de las certificaciones aplicadas ha permitido un fortalecimiento de la competitividad 0 ha llevado a procesos de normalización que deterioran la diferenciación del producto?

En la sección 2 de este trabajo se aborda el entorno en el que se inscriben los procesos de certificación. Posteriormente, se presenta en la sección 3 una aproximación a las realidades por vía de lo observado por las organizaciones de pequeños productores agrícolas. Finalmente, se presentan las conclusiones obtenidas en este estudio.

\section{El entorno de las certificaciones}

\subsection{Las dinámicas de las certificaciones y su reflejo en Costa Rica}

Hatanaka y Busch (2008) destacan la tendencia en la aplicación de regulaciones ambientales y sociales a darle un espacio de mayor relevancia a los distintos actores no estatales, en la cual pasa del estado de la supervisión directa y el control de las regulaciones, a una función más indirecta de control a las partes interesadas y de fomento de la autorregulación de sus propias actividades. En el caso de la alimentación y la agricultura, se ha buscado mantener las regulaciones pero de una manera menos costosa. El resultado es que se han fortalecido los sistemas de certificaciones, en los cuales actores no estatales, tales como las cadenas de minoristas y las ONG, alcanzan un papel creciente y más activo en la elaboración de normas (Hatanaka y Busch, 2008, pág. 76). 
Un recuento de Díaz y Avendaño (2014), que se presenta en el cuadro 1, señala que en Costa Rica predominan los sistemas de etiquetado tipo I (creados por organismos independientes que no intervienen en el mercado), los cuales tienden a normalizar y a fortalecer su credibilidad ante el consumidor. Estos etiquetados pueden ser orientados al consumidor o formar parte de los requerimientos de las empresas compradoras (modelo "business to business") (Tallontire, Dixon, y Benton, 2012, pág. 101).

Cuadro 1

Sistemas de certificación ambiental presentes en Costa Rica

\begin{tabular}{|c|c|c|c|}
\hline Tipo de Eco Etiquetas & $\begin{array}{l}\text { Sellos de empresas } \\
\text { individuales }\end{array}$ & $\begin{array}{l}\text { Certificaciones } \\
\text { nacionales } \\
\text { colectivas }\end{array}$ & $\begin{array}{l}\text { Certificaciones } \\
\text { colectivas } \\
\text { internacionales }\end{array}$ \\
\hline $\begin{array}{l}\text { Tipo I } \\
\text { Creadas por un organismo } \\
\text { independiente que no interviene } \\
\text { en el mercado }\end{array}$ & $\begin{array}{l}\text { GlobalG.A.P. 1/ } \\
\text { C.A.F.E. (Starbucks) } \\
\text { AAA Nesspreso }\end{array}$ & $\begin{array}{l}\text { Esencial } \\
\text { Rica }\end{array}$ & $\begin{array}{l}\text { Organic; } \\
\text { Rainforest } \\
\text { Alliance; } 4 \text { C } \\
\text { common code; } \\
\text { ISO 50001; ISO } \\
14000\end{array}$ \\
\hline $\begin{array}{l}\text { Tipo I equivalente } \\
\text { sistemas de normas } \\
\text { individuales } 0 \text { múltiples } \\
\text { orientadas a la sostenibilidad } \\
\text { de ciertas etapas de la } \\
\text { producción }\end{array}$ & & $\begin{array}{l}\text { Bandera } \quad \text { Azul } \\
\text { Ecologica. }\end{array}$ & $\begin{array}{l}\text { Certificación } \\
\text { comercio justo; } \\
\text { Amigable con los } \\
\text { pájaros } \\
\text { (Smithsoniano) }\end{array}$ \\
\hline $\begin{array}{l}\text { Tipo II } \\
\text { Auto declaraciones de los } \\
\text { propios fabricantes } \\
\text { /productores }\end{array}$ & & $\begin{array}{l}\text { Sistema de Gestión } \\
\text { Socio ambiental } \\
\text { para la producción } \\
\text { sostenible de la } \\
\text { piña (SG-PSP) }\end{array}$ & CLAC (PPP) \\
\hline $\begin{array}{l}\text { Tipo III } \\
\text { Auto declaración detallada del } \\
\text { producto (ciclo de vida) }\end{array}$ & Universidad EARTH & & \\
\hline
\end{tabular}

1/ No directamente visibles para los consumidores (de empresa a empresa)

Fuente: Díaz- Porras y Avendaño-Escudero, 2014, pág. 21.

A partir del cuadro 1 (Díaz-Porras y Avendaño-Escudero, 2014, págs. 22-23) se puede concluir que:

- Predominan los sistemas internacionales (tipo I o II) que han evolucionado como requerimientos desde el comprador en las cadenas de valor. 
- En Costa Rica se han planteado iniciativas gubernamentales que buscan crear sostenibilidad, a través de la creación de ventajas competitivas directamente en los mercados (caso Costa Rica esencial), así como mediante mejoramiento de los procesos productivos frente a las comunidades (caso bandera azul).

Asimismo en el cuadro 1 se muestran dos iniciativas desarrolladas desde los productores, una de carácter internacional en comercio justo, (el sello de pequeños productores) que busca la diferenciación del producto en relación con el tipo de productores. La otra es de carácter gremial, para contrarrestar los cuestionamientos acerca de los impactos ambientales de la producción de piña.

\subsection{La plataforma de apoyo institucional en Costa Rica ${ }^{5}$}

Un aspecto de mucha relevancia en el entorno para operar con certificaciones lo constituye la existencia y tipo de funcionamiento de la plataforma de apoyo para las organizaciones que adoptan una estrategia de diferenciación de productos.

El análisis se realiza a través de un mapeo de las organizaciones participantes de la plataforma de apoyo, el tipo de apoyo que realizan, y los vínculos que aparecen en el sistema que conforman. Se distinguen cinco tipos de organizaciones:

1. Empresas y organizaciones de las certificaciones

2. Empresas y organizaciones certificadoras

3. Empresas y organizaciones reguladoras

4. Organizaciones e instituciones públicas

5. Empresas, organizaciones e instituciones privadas

Por su parte, estas organizaciones desarrollan diferentes tipos de apoyos, que clasificamos en cinco tipos:

- Apoyo económico y fiscal: se entiende tanto subsidios como préstamos, créditos y también la disminución o ausencia de pagos fiscales.

- Apoyo técnico: es la forma de apoyo y fomento más amplia. Este tipo de apoyo incluye capacitaciones para temas ambientales, financieros y administrativos entre otros, servicio de asesoría, manuales, E-Learning y transferencia de tecnología, para mencionar algunos.

\footnotetext{
${ }^{5}$ Este apartado se basa en los resultados del trabajo de graduación de la estudiante Susanne Ratge del Bachillerato en Administración de Empresas de la Universidad de Münster, Alemania.
} 
- Apoyo estructural: consiste básicamente en investigaciones que ejecutan las diferentes organizaciones para mejorar el entorno del productor y obtener más conocimiento sobre temas relacionados con la agricultura sostenible, e inversiones en infraestructura. Por esto, el apoyo estructural es principalmente un apoyo indirecto para los productores.

- Apoyo de comercialización: incluye servicios, por un lado, a los productores tales como servicios financieros, servicios de plataformas, plazas de mercado, espacios de presentación, así como servicio de bases de datos; y por otro lado, para los empresas y organizaciones como compradores de la mercancía y como oferentes de servicios financieros, por ejemplo.

- Apoyo de intercambio interno: fomenta el intercambio de conocimiento y experiencias entre los productores. Tal objetivo se intenta obtener a través de la organización de eventos de encuentro para, por ejemplo, productores orgánicos, foros y otros encuentros.

1.3 Relaciones entre los actores del entorno de las certificaciones del tipo I y tipo I similar

Para efectos de analizar las relaciones entre los actores, se considera, en primer lugar, una aproximación de la intensidad del apoyo brindado a las organizaciones, que luego permite establecer una clasificación de los diferentes tipos de organizaciones por la intensidad del apoyo que ofrecen y las vinculaciones entre los diferentes tipos de organizaciones, así como evidenciar las relaciones entre ellas.

En la figura 1 se muestra que las organizaciones privadas tienen el mayor peso en las acciones de apoyo. En menor proporción, se tienen las organizaciones de las certificaciones y las públicas. 


\section{Figura 1}

Costa Rica. Participación de las actividades de apoyo según tipo de organizaciones: Certificaciones tipo I y I similar (2014)

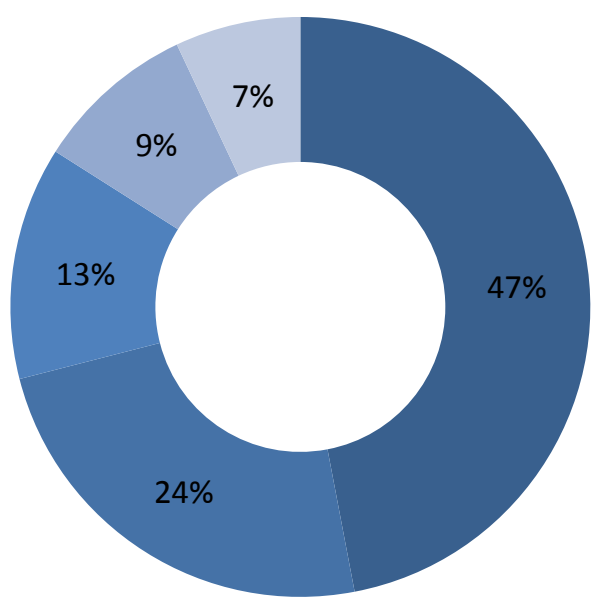

- Org. privadas

- Org. de certificación

- Org. Públicas

Org.certificadores

Org.reguladores

Fuente: Elaboración propia

La intensidad del apoyo se estima considerando solo al apoyo directo según los cinco tipos definidos anteriormente. El apoyo de estabilización y regulación del sistema que ofrecen los reguladores no se encuentra incluido en este término, por lo que se va a señalar posteriormente de otra manera. Por ende, para calcular el orden de los tipos de organizaciones respecto a la intensidad de su apoyo, no se toman en cuenta las organizaciones reguladoras.

La intensidad del apoyo se define por la proporción de las acciones de apoyo directo respecto a las acciones no ofrecidas por organización6; es decir, por cuántos tipos en promedio fomenta cada clase de organización. No se consideran las organizaciones reguladoras que no brindan ningún tipo de apoyo.

En la figura 2 se muestran los resultados del indicador que dimensiona la cantidad de acciones de apoyo por tipo de organización. Los resultados de este cálculo muestran que las organizaciones públicas ofrecen en promedio 2,5 tipos de apoyo por organización, las organizaciones privadas 1,75, las organizaciones de las certificaciones 1,57 y las organizaciones certificadores 1,33 tipos de apoyo por organización.

\footnotetext{
${ }^{6}$ Intensidad de apoyo se define como la proporción $\mathrm{I}_{\mathrm{a}}=$

$\sum_{i}^{n}$ Acciones de apoyo ofrecidas $/ \sum_{i}^{n}$ Acciones de apoyo no ofrecidas, ${ }^{\prime}$ : tipo de organización.
} 
Figura 2

Costa Rica. Indicador de intensidad de apoyo por tipo de organizaciones de la plataforma de apoyo, certificaciones tipo I y I similar. 2014

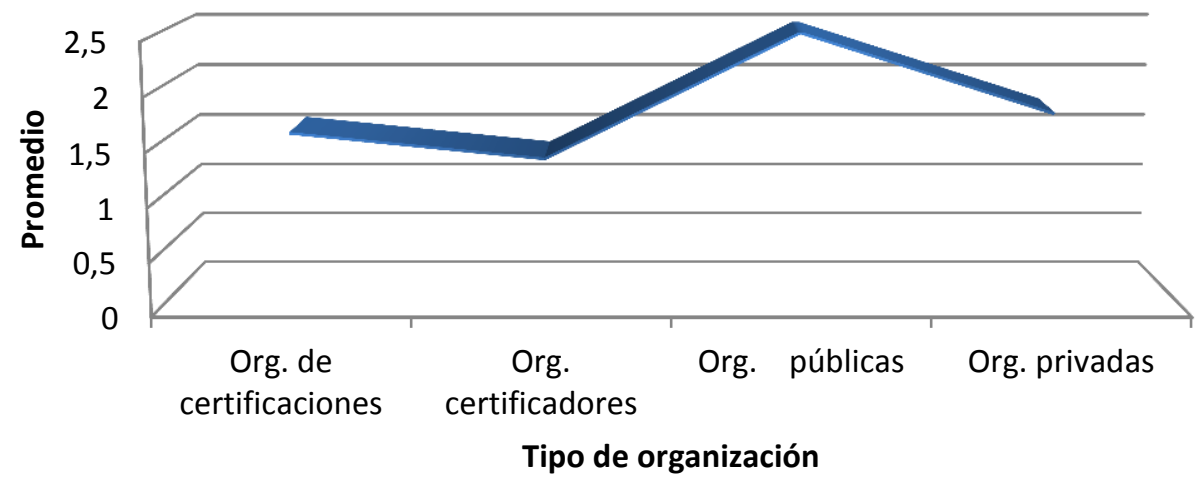

Fuente: Elaboración propia

Por lo tanto, las organizaciones públicas brindan apoyo de mayor intensidad y los certificadores de menor intensidad.

Otra aproximación se presenta en la figura 3, en la cual se observan los tipos de apoyo que se presentan con mayor frecuencia. Según los cálculos realizados, el $46 \%$ de las acciones de apoyo que realizan las organizaciones son del tipo técnico. Eso se puede explicar por los cambios que surgen de la implementación de nuevos procesos productivos y administrativos. En segundo lugar, con el $20 \%$ de las acciones, se tiene el apoyo a la comercialización. Las acciones de apoyo estructural y de intercambio interno significan el $12 \%$ de las acciones, mientras que con un porcentaje algo menor del $10 \%$ se ubica el apoyo económico, que representa el menos frecuente en el entorno de las certificaciones de los tipos I y I similar. 
Figura 3

Costa Rica. Tipos de apoyo en las certificaciones tipo I y I similar por porcentaje. 2014

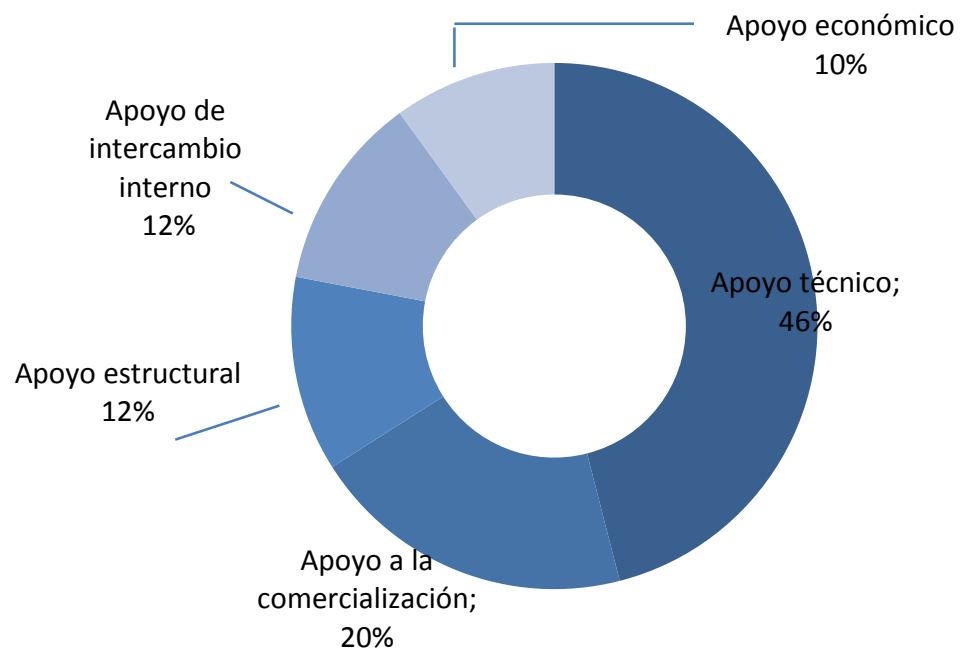

Fuente: Elaboración propia

En la figura 4 se pueden observar el tipo de relaciones que se dan entre estas organizaciones.

Figura 4

Costa Rica. Relaciones entre las organizaciones de apoyo en las certificaciones tipo I y I similar (2014)

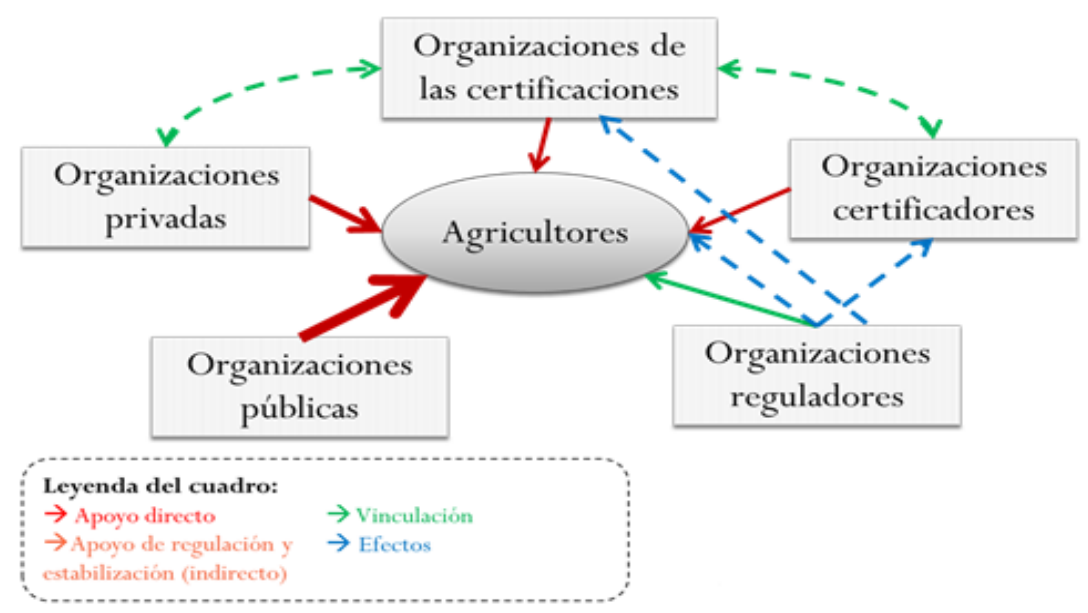

Fuente: Elaboración propia. 
Las flechas continuas muestran relaciones con más del $50 \%$ de las organizaciones de productores y cuyo grosor se relaciona con la intensidad del apoyo. Las flechas discontinuas muestran relaciones entre las organizaciones de la plataforma de apoyo con menos del $50 \%$ de las organizaciones, las cuales pueden ser de vinculación o de efecto

\subsection{Relaciones entre los actores de las certificaciones del tipo II}

En la categoría del tipo II se analizan dos sellos diferentes cuyas relaciones se visualizan en la figura 5. Debido a que las certificaciones de esa categoría tienen como característica la autodeclaración por los productores, es más difícil encontrar un sistema para todas las certificaciones. En este caso se intenta incluir las características de los dos sellos analizados: Pequeños productores para el comercio justo y el Sistema de Gestión Socio Ambiental para la Producción Sostenible de la Piña (SG PSP).

Figura 5.

Costa Rica. Relaciones entre los actores de las certificaciones del tipo II (2014)
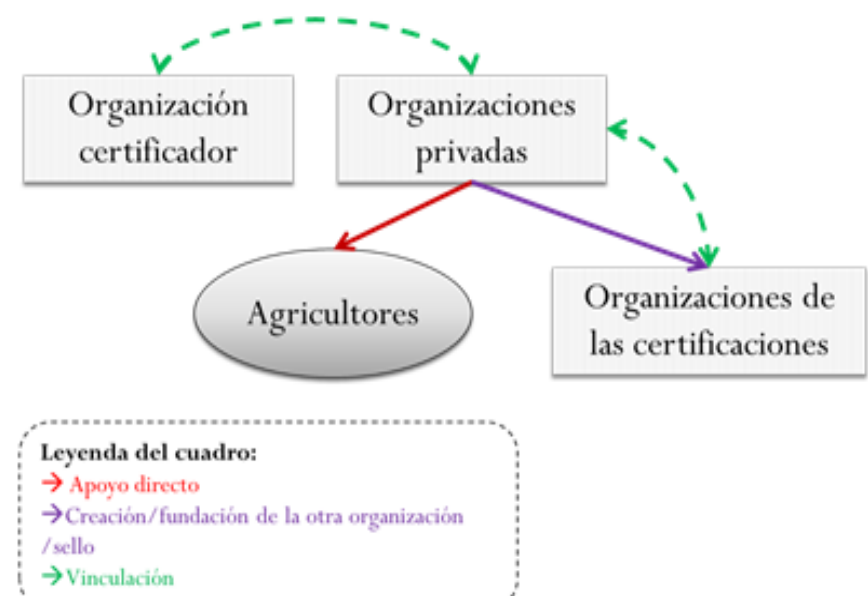

Fuente: Elaboración propia

En el entorno de los agricultores certificados en el tipo II se encuentran tres actores: las organizaciones privadas, las de las certificaciones y las certificadoras. En los dos casos analizados, una organización privada fundó el sello respectivo y no lo originó una entidad independiente, lo que lo hace diferente de las certificaciones del tipo I. La fundación implica una fuerte vinculación pues la fundadora administra el sello. 
En la figura 5 también se visualiza el apoyo directo y las vinculaciones, considerando la relación de la fundación o creación que se expresa en el vínculo entre las organizaciones privadas y los agricultores. Una de las funciones de las organizaciones privadas es la autorización de los certificadores y seguir verificando su desempeño.

En este conjunto se encuentra también la relación de apoyo directo, que es ofrecido por las organizaciones privadas a los productores. En el caso del SPP, son la FUNDEPPO y la CLAC. Para el sello del SG PSP son la CANAPEP y la COSAP. En la investigación no se puedo ahondar en las relaciones con y de las organizaciones certificadoras.

Resulta llamativo que existen más vinculaciones entre los actores en el sistema de las certificaciones del tipo I y I similar. Eso se puede explicar por la falta de organizaciones públicas y reguladoras. Asimismo, un aspecto muy importante es la fundación del sello por una de las organizaciones privadas, por su característica especial de autodeclaración y por eso impulsado por los actores más cercanos.

\subsection{Relaciones entre los actores de las certificaciones tipo III}

En esta categoría se incluye el caso de la certificación Carbono Neutralidad. La relación de mayor importancia, tal como se observa en la Figura 6, la tiene la organización de la certificación con su certificador, que en este caso es la Universidad EARTH. Dicha organización ofrece el servicio de la verificación y certificación, y también brinda el apoyo técnico.

Figura 6.

Costa Rica. Relaciones entre los actores de las certificaciones del tipo III ( 2014)

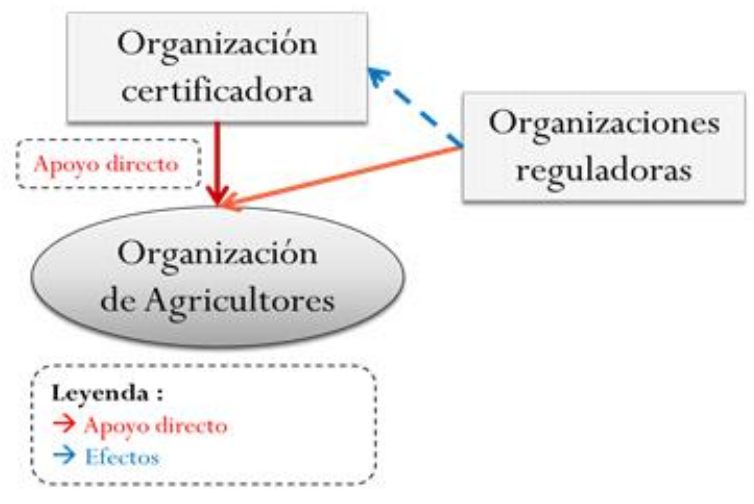

Fuente: Elaboración propia 


\section{Las realidades}

La situación de las organizaciones de productores certificadas se aproximan a partir de los resultados de una encuesta, cuyos resultados se documentan en Díaz, Delgado y Páez (2015). Los datos fueron obtenidos mediante entrevista a gerentes de organizaciones de pequeños productores agrícolas durante el primer semestre del 2014, los cuales se exponen a continuación.

\subsection{Características de las organizaciones certificadas}

Las organizaciones de productores agrícolas certificadas que se entrevistaron, se concentran en la actividad cafetalera. En la figura 7 se presentan sus características generales.

\section{Figura 7.}

Costa Rica. Organizaciones de pequeños productores certificadas: Características generales. 2014

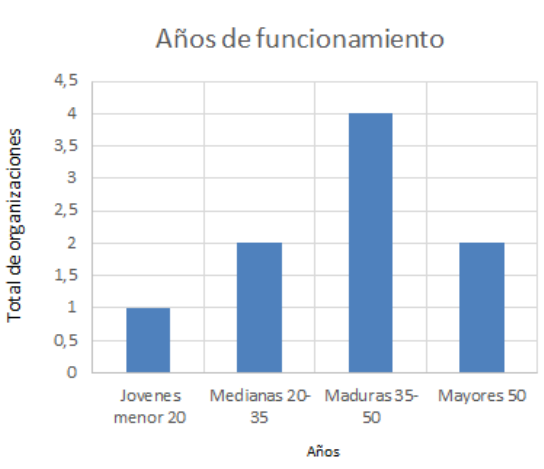

Distribución por cantidad de productores

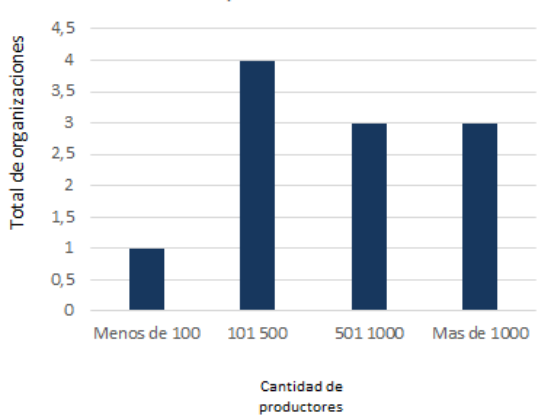

Fuente: Díaz, Páez y Delgado, 2015

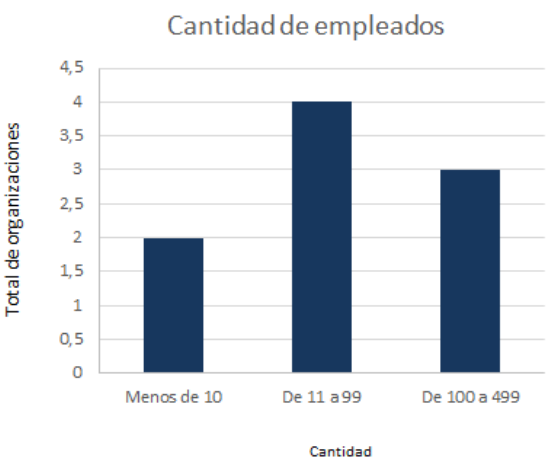

Distribución por el tamaño promedio de las fincas

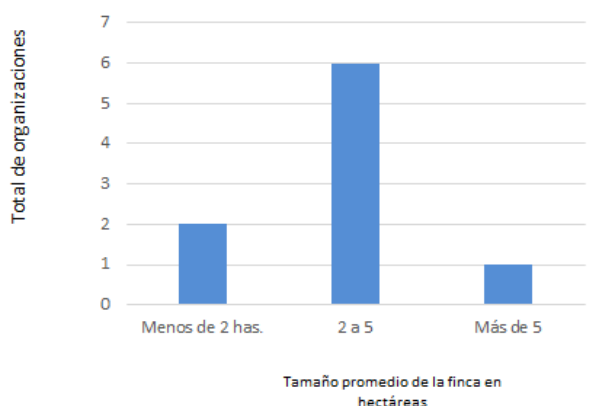


De las organizaciones entrevistadas, las de banano y café orientan sus productos principalmente a la combinación de los mercados internacionales y nacionales. La producción de mora orgánica representa el único caso en el que su producción se orienta estrictamente al mercado local, pues el precio en el mercado internacional no le es atractivo y la de azúcar de caña es la única que se orienta exclusivamente al mercado internacional.

\subsection{Sistemas de certificación}

En la encuesta se detectaron 11 sistemas de certificación, la mayor parte son de tipo I, es decir, desarrolladas por organismos independientes y acogidos por los compradores, en sus requerimientos de compra (figura 8).

Figura 8

Costa Rica. Certificaciones y organizaciones certificadas. 2014
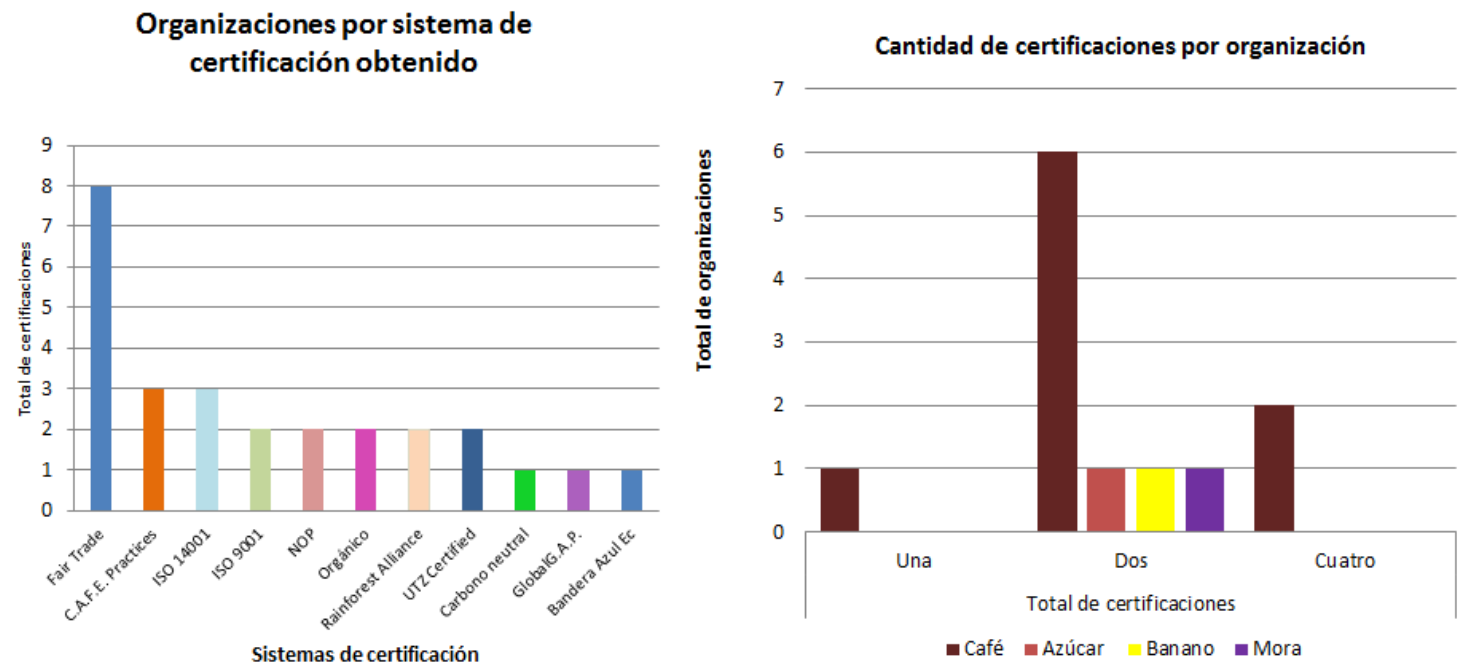

Fuente: Díaz, Páez y Delgado, 2015 


\subsection{Desarrollo de factores de competitividad}

El vínculo que ha interesado en este estudio entre las certificaciones y el desarrollo de factores de competitividad con la anterior evidencia permite concluir que las organizaciones, una vez certificadas, han ingresado a mercados de mayor exigencia y calidad, mas no ha implicado automáticamente mejores precios. Esto evidencia que un primer logro es haber obtenido un acceso de mercado, pues para una parte importante de las organizaciones entrevistadas sigue siendo el precio el factor clave al vender. De esta forma, la diferenciación obtenida con las certificaciones no alcanza plenamente su impacto en el mercado.

Un aspecto complementario respecto a la utilidad de las certificaciones en el mercado lleva a verificar cuáles características del producto le interesa o preocupa más al comprador. El resultado es que la mayor cantidad de respuestas (35\%) se refieren al precio. Sin embargo, está acompañada por otras preocupaciones tales como las condiciones en que se da el proceso productivo (el medio ambiente -25\%- y las condiciones sociales - $25 \%$-). Este resultado es consistente con el tipo de posicionamiento del producto que está apelando a convenciones ${ }^{7}$ de tipo ético de los compradores.

Mayor competitividad puede, sin embargo, ser alcanzada por vía del mejoramiento en el manejo de los costos y cambios en el proceso productivo. Las respuestas en las entrevistas, principalmente en la organización de productores de café, muestran dos cambios relevantes, referidos al cambio de los insumos y la preparación de la tierra; los cuales junto al mejor manejo administrativo, son los ajustes más importantes en el proceso productivo (figura 9).

\footnotetext{
${ }^{7}$ En referencia a las convenciones de calidad incorporadas en las decisiones de los consumidores y que se vinculan con la gobernanza de la cadena (Ponte \& Gibbon, 2005, págs. 18-20), se distinguen convenciones industriales, ligadas a la calidad; convenciones cívicas, que se orientan a satisfacer demandas de los consumidores relacionadas con sus preocupaciones acerca de las implicaciones sociales o ambientales en que se desarrollan los procesos productivos, y convenciones de lealtad, en las cuales los rasgos distintivos (de origen) son fundamentales a partir de interacciones repetidas.
} 


\section{Figura 9}

Organizaciones certificadas: Opciones de cambio y su intensidad en los procesos productivos. 2014.

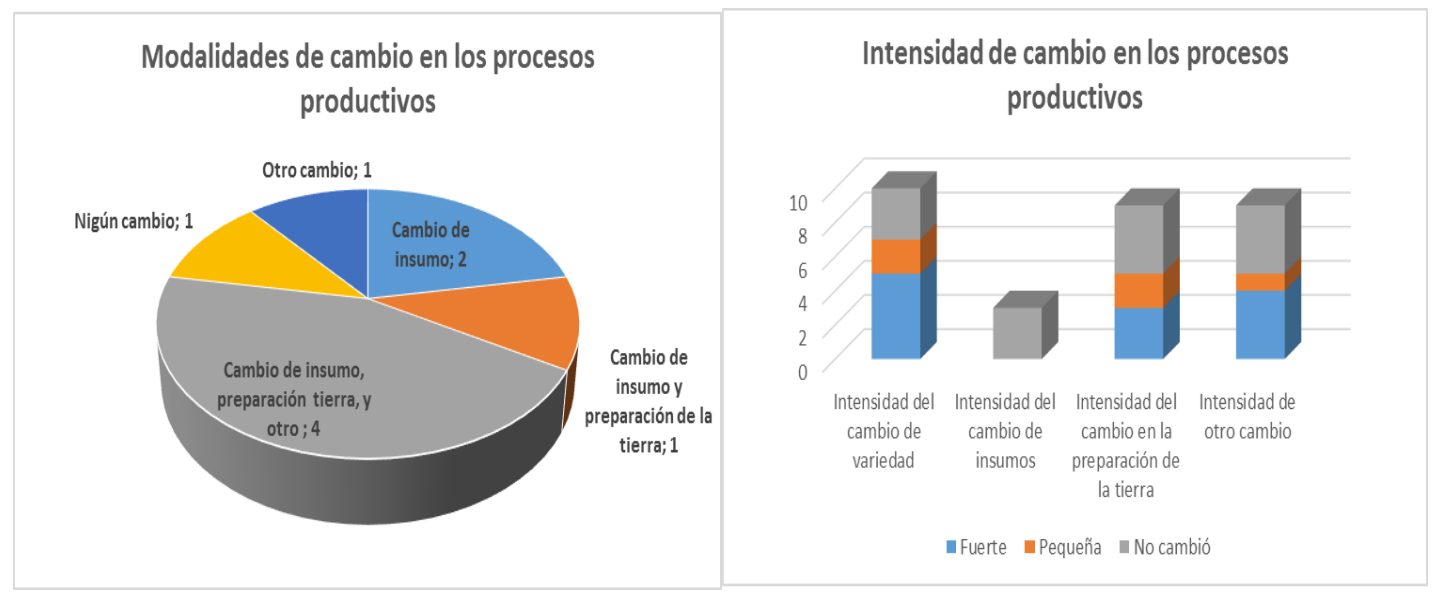

Fuente: Díaz, Páez y Delgado, 2015, pág.15.

\subsection{Impactos de las certificaciones}

Como reflejo de lo anterior, una tercera parte de las organizaciones, todas cafetaleras, indicó que el costo de producción aumentó, en tanto que los demás consultados dan respuesta negativa. Se menciona con insistencia el aumento en los costos de producción, tanto en el proceso productivo como en lo administrativo.

Por lo tanto las certificaciones no tienen impactos claros sobre la productividad en los productos analizados, aunque implican mayores costos. Ante ello una pregunta relevante refiere a las dificultades enfrentadas en el proceso de certificación. En ese sentido las barreras que se mencionan con mayor frecuencia se refieren a la tramitología y los costos asociados con la certificación. Con menor grado de dificultad se tienen la barrera cultural y las opciones tecnológicas, en este orden. Sin embargo la barrera cultural es también muy señalada por los gerentes, por lo cual adquiere un nivel de mucha relevancia en los procesos de certificación. 


\subsection{Sostenibilidad de las certificaciones y competitividad}

Como reflejo de lo anterior, una tercera parte de las organizaciones, todas cafetaleras, indicó que el costo de producción aumentó, en tanto que los demás consultados dan una respuesta negativa. Se menciona con insistencia el aumento en los costos de producción, tanto en el proceso productivo como en lo administrativo.

Por lo tanto, las certificaciones no tienen impactos claros sobre la productividad en los productos analizados, aunque implican mayores costos. Ante ello, una pregunta relevante refiere a las dificultades enfrentadas en el proceso de certificación. En ese sentido, las barreras que se mencionan con mayor frecuencia se refieren a la tramitología y los costos asociados con la certificación. Con menor grado de dificultad, se tienen la barrera cultural y las opciones tecnológicas, en este orden. Sin embargo, la barrera cultural es también muy señalada por los gerentes, por lo cual adquiere un nivel de mucha relevancia en los procesos de certificación.

Figura 10.

Trayectoria de certificación en las organizaciones entrevistada. 2014

\begin{tabular}{|c|c|c|c|c|c|c|c|c|c|c|c|c|c|c|c|c|c|c|c|c|}
\hline Organización & $1988 \ldots$ & $1990 \ldots$ & 97 & 98 & 99 & 2000 & 1 & 2 & 3 & 4 & 5 & 6 & 7 & 8 & 9 & 10 & 11 & 12 & 13 & 14 \\
\hline \multirow{2}{*}{1 Café } & \multicolumn{20}{|c|}{ FAIR TRADE } \\
\hline & & & & & & & & & & & \multicolumn{10}{|c|}{ ORGÁNICO } \\
\hline 2 Café & & \multicolumn{19}{|c|}{ FAIRTRADE } \\
\hline 3 Azúcar & & \multicolumn{19}{|c|}{ FAIRTRADE } \\
\hline A Cafó & & \multicolumn{19}{|c|}{ FAIRTRADE } \\
\hline 4 caje & & & & & & & & & & \multicolumn{11}{|c|}{ C.A.F.E. Practices } \\
\hline \multirow{2}{*}{5 Banano } & & & & & \multicolumn{16}{|c|}{ FAIR TRADE } \\
\hline & & & & & & \multicolumn{15}{|c|}{ GLOBAL GAP } \\
\hline \multirow{2}{*}{6 Café } & & & & & & \multirow{2}{*}{\multicolumn{4}{|c|}{ ISO $14001+$ UTZ }} & \multirow{2}{*}{\multicolumn{5}{|c|}{ C.A. F.E. Practices }} & \multirow{2}{*}{\multicolumn{6}{|c|}{ FAIR TRADE }} \\
\hline & & & & & & & & & & & & & & & & & & & & \\
\hline 7 Café & & & & & & & & & & \multicolumn{11}{|c|}{ ISO 14001 + C.A.F.E. Practices + FAIR TRADE + RFA + UTZ } \\
\hline \multirow{2}{*}{8 Café } & & & & & & & & & & & \multicolumn{10}{|c|}{$\begin{array}{l}\text { FAIR TRADE } \\
\end{array}$} \\
\hline & & & & & & & & & & & & & & & \multicolumn{6}{|c|}{ Rain Forest Alliance } \\
\hline \multirow{2}{*}{9 Café } & & & & & & & & & & & & & & & & & \multicolumn{4}{|c|}{ CARBONO NEUTRAL } \\
\hline & & & & & & & & & & & & & & & & & & & ANDE & ERA \\
\hline \multirow{2}{*}{10 Mora } & & & & & & & & & & & & & & & & & \multicolumn{4}{|c|}{ Organico } \\
\hline & & & & & & & & & & & & & & & & & & & & NOP \\
\hline
\end{tabular}

Fuente: Díaz, Páez y Delgado, 2015, pág.17

Una garantía, para las organizaciones que exportan, la constituye el hecho de que la certificación les ha avalado su permanencia en esos mercados, a través de la competitividad lograda por las organizaciones, que se manifiesta en su participación internacional (figura 11), al exponerse en un ambiente más exigente y competido. Sin embargo, como contraposición, se tiene que muchas organizaciones participan en el mercado nacional, lo que las fortalece al diversificar riesgos y el desarrollo de aprendizajes en productos de mayor valor agregado, como lo denota el caso del café. La producción de azúcar se concentra en el mercado internacional en el nicho del comercio justo, con la limitante para organizaciones individuales de que no pueden desarrollar otros 
productos, pues se tiene una organización sectorial a través de LAICA en su carácter de industrializador único ${ }^{8}$.

Figura 11.

Ventas al extranjero de las organizaciones de pequeños productores certificadas en porcentajes. 2014

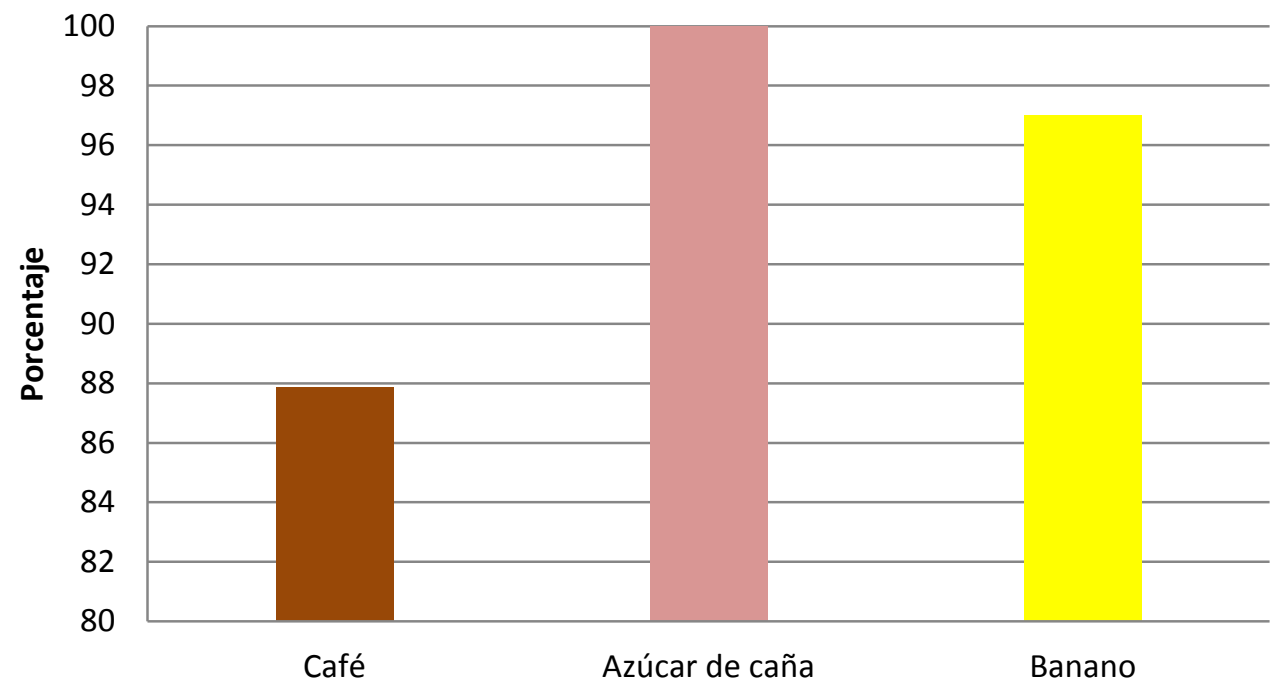

Fuente: Díaz, Páez y Delgado, 2015, pág 19.

En relación con los elementos que afectan a las organizaciones certificadas, destacan como desventajas de las certificaciones básicamente dos aspectos: por una parte, el proceso de implementación mismo que implica gastos y presión a la organización; por otra parte, se tiene la tramitología asociada con el logro y mantenimiento de las certificaciones, esto es mencionado principalmente por las organizaciones de productores de café.

Como contraparte, en cuanto a las ventajas percibidas por las organizaciones, se destacan el factor precio, junto a las ventajas derivadas en términos de mejoras de procesos, mejoras ambientales y, con ello, mejoras competitivas.

\footnotetext{
${ }^{8}$ Según la ley 7818, Ley Orgánica de la Agricultura e Industria de la Caña de Azúcar, se define como una corporación no estatal para el ejercicio de actividades de comercialización y de las demás de carácter empresarial que le asigna esta ley. Las actividades de comercialización se definen como: comercializar alcohol, azúcar, mieles y otros subproductos de la industrialización de la caña de azúcar.
} 
En relación con el acceso a nuevos mercados o mercados con más exigencia, se observa que solo en el caso del café los gerentes indican que se ascendió a un mercado más exigente, lo cual refleja la evolución del mercado con énfasis en la calidad; mientras que para los otros productos no hay variantes.

Es interesante que al poner atención a las modificaciones se tiene la exigencia de volúmenes mínimos de entrega, generando dificultades por falta de financiamiento en los productos azúcar de caña, banano y mora.

Es de esperar que la diferenciación de productos mediante certificaciones lleve a las organizaciones a una mayor exposición en el mercado. Una aproximación se tiene en relación con los medios de mercadeo. El resultado obtenido muestra que la mayor parte de las organizaciones no cuenta con departamento de mercadeo. Resulta interesante que las que lo tienen son organizaciones de productores de café, en cuya actividad la diferenciación de productos es más generalizada y por lo tanto el esfuerzo de ventas requiere de mayor espacio.

En un sentido más sintético, las opiniones de los gerentes entrevistados acerca del comportamiento del mercado son positivas, pues los mercados crecen o al menos se mantienen sin cambio.

\subsection{Perspectivas de las organizaciones y su participación en las cadenas de valor}

Un primer aspecto a destacar refiere a las posibilidades de las organizaciones de valorizar su esfuerzo de certificación en el contexto de las cadenas en que participan. Con referencia al marco fundamentado por Lee, Gereffi y Bauvais (2012) citado en Díaz et al. (por publicar, pág. 7), se tiene que en general las cadenas en que participan las organizaciones incluidas en el estudio son cadenas con gobernanza desde el comprador, en las cuales inciden decisivamente las cadenas de supermercados, que son muy propensas a que las certificaciones se traduzcan en requisitos de acceso (Bingen y Busch, 2006, págs. 9-10). Sin embargo, en el caso de las organizaciones participantes del Comercio Justo, en la medida de que parte de su producción va al mercado por mecanismos de coordinación alternos, que les acerca al comprador directamente, logran posicionarse de mejor forma al diferenciar su producto.

En sus proyecciones hacia el futuro, las organizaciones visualizan mantenerse con las certificaciones. En las entrevistas se hizo un ejercicio prospectivo, al indagar sobre las estrategias que se plantean las organizaciones hacia adelante. Con relación a los medios de mercadeo por parte de las organizaciones, una vez que han obtenido su certificación, se determinó que las organizaciones cafetaleras visualizan en los eventos y medios de comunicación sus esfuerzos, mientras que en la actividad cañera es este último medio el que se percibe. 
En lo referente a las certificaciones mismas, las respuestas apuntan en varias líneas (figura 15). Se percibe implícita o explícitamente la estrategia de seguir con las certificaciones, mas con variantes para mejorar al interior de la organización con información a los productores, la incorporación de nuevas certificaciones y proyectarlas en sus actividades de mercadeo y promoción.

Figura 12

Costa Rica. Organizaciones certificadas: estrategias a futuro respecto a las certificaciones. 2014

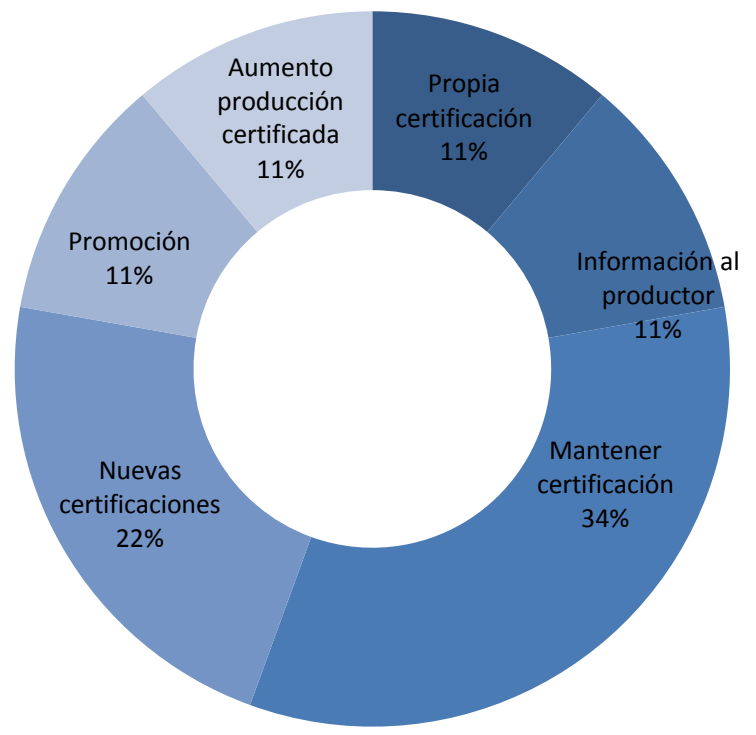

Fuente: Díaz, Páez y Delgado, 2015, pág.24

\section{Conclusiones}

Un primer resultado del análisis es que en el mercado predominan las certificaciones del tipo I, es decir, las creadas por un organismo independiente que no intervienen directamente en el mercado y orientadas a la sostenibilidad de ciertas etapas de la producción.

Desde la incorporación del sello del comercio justo, en 1988, se han ido incorporando otros sellos, lo cual amplía las opciones para tratar de diferenciar los productos. La necesidad de certificarse para poder acceder a ciertos nichos de mercado ha generado recientemente que algunas organizaciones posean desde el inicio de sus actividades varios sellos. 
Para los agricultores participantes en las organizaciones, los procesos de certificación han significado un costo, aspecto que ha sido asimilado paulatinamente. Sin embargo, se mencionan obstáculos importantes en su implementación, tanto en el proceso administrativo (no estaban acostumbrados a ciertos controles que ahora deben cumplir), como en el proceso productivo. Asimismo, se plantea como obstáculo la cultura (costumbre) de los productores y la tramitología que se genera para la aprobación del sello. Importante ha sido que el proceso productivo en definitiva cambió, pues los entrevistados muy claramente manifestaron que la forma de sembrar varió, unos más radicales que otros, pero para cumplir con los requisitos. Sin embargo, quedó claro que no existe un aumento significativo en los rendimientos agrícolas.

La calidad sí fue objeto de mejora pues se determinó que las certificaciones han significado una opción real para poder acceder a ciertos nichos ambientalmente amigables del mercado nacional e internacional, sin embargo, aunque la mayoría de los sellos no garantizan mejores precios, el uso de estos ha dado mayor empoderamiento a las organizaciones frente a sus compradores y competidores.

Se puede afirmar que la certificación ha permitido un mejor posicionamiento y, por consiguiente, mayor estabilidad en la cadena de valor. Sin embargo, es importante tener en consideración los peligros de saturar el nicho de mercado, por la atracción de nuevos productores, resultado de la nueva cultura de protección del medio ambiente.

En relación con las expectativas de las organizaciones, una tercera parte menciona continuar con la certificación y una quinta parte planea incorporar otra certificación. Esto muestra la importancia y el valor agregado, en términos de marca, que presenta la utilización de las certificaciones.

Como corolario, al reflexionar sobre los requerimientos de políticas se pueden distinguir las siguientes áreas críticas:

Necesidad de un mayor involucramiento de productores en los procesos de certificación. Faure et al. han planteado esta debilidad pues en las organizaciones, desde de su adopción, las certificaciones se perciben como un asunto de los dirigentes (Faure y otros, 2014).

- El diseño de políticas debe abarcar el soporte para enfrentar la evolución de nuevas normas, nuevos y diversos requerimientos, incorporación de nuevas certificaciones, etc.

- Apoyar el acceso de pequeños productores a certificaciones mediante la organización.

- Afrontar la realidad de las certificaciones en las estructuras de gobernanza de la cadena, pues la certificación no garantiza automáticamente beneficios económicos. Esto hace eco de la preocupación planteada por Bitzer, Francken y Glasbergen (2009, pág. 143) en el sentido de que los gobiernos de los países productores de café han estado desconectados de las alianzas en las cadenas, sin un enfoque estratégico. 
- Promover el mercado interno como una opción para los productores certificados, distinguiendo las barreras para su desarrollo.

\section{Bibliografía referenciada}

Abarca, R., y Sepúlveda, S. (2001). Eco-etiquetado: Un Instrumento para Diferenciar Productos e Incentivar la Competitividad. Serie Cuadernos Técnicos IICA(17). San José, Costa Rica.

Auld, G. (2011). Evaluación de la certificación como gobernabilidad: efectos y consecuencias ampliadas para el café. Ensayos Sobre Economía Cafetera (27). Recuperado el 05 de 09 de 2013, de http://www.federaciondecafeteros.org/static/files/evaluacion_certificacion_gobernab i

Bingen, J., y Busch, L. (2006). Introducción. En L. Busch, y J. Bingen (Edits.), Agricultural Standards. The Shape of the Global Food and Fiber Sytem. The Netherlands: Springer.

Bitzer, V., Francken, M., y Glasbergen, P. (2009). Alianzas Intersectoriales para una cadena de café sostenible: ¿ Te-niendo realmente en cuenta la sostenibilidad o solamente recogiendo las cerezas del café ? Ensayos Sobre Economía Cafetera, 25, 123-145. Ensayos Sobre Economía Cafetera,(25), 123-145.

Díaz Porras, R., y Avendaño Escudero, M. (2014). Evolución de las certificaciones ambientales: perpectivas para el mejoramiento competitivo de los pequeños productores agrícola en Costa Rica. Cuadernos de Política Económica(002-2014). ). doi:ISSN: 2215-4159,

Díaz, R., Delgado, A., y Páez, W. (2015). Certificaciones y competitividad de las organizaciones de pequeños productores: Alguna evidencia empírica. Cuadernos de Política Económica(001-2015). Obtenido de http://hdl.handle.net/11056/10886

ETC GROUP. (2012).¿ Quién controlará la economía verde ? Ottawa, Ontario. Recuperado el 3 de Setiembre de 2014, de http://www.etcgroup.org/files/ETC_wwctge_ESP_v4Enero19small.pdf

Faure, G., Cocq, J., Vagneron, I., Hocdé, H., Soto, G., y Kessari, M. (2014). Estrategias de organización de productores de cafè en Costa Rica con respecto a las certificaciones ambientales y sociales. En J. Cocq, F. Sáenz, y G. Faure,. En J. Cocq, F. Sáenz, y G. Faure (Edits.), Globalización y desafíos para la pequeña agricultura en Costa Rica: experiencia de organización y generación de servicios para el acceso a mercados. Heredia: EUNA.

Hatanaka, M., y Busch, L. (2008). Third-Party Certification in the Global Agrifood System: An Objective or Socially Mediated Governance Mechanism? Sociología Ruralis, 48(1). Obtenido de http://onlinelibrary.wiley.com/doi/10.1111/j.14679523.2008.00453. 
Liu, P. (2010). Retrieved from http://www.cabdirect.org/abstracts/20103145023.html. En J. Albert (Ed.), Innovations in food labelling. Oxford: FAO and Woodhead Publishing Limited.

Ponte, S., y Gibbon, P. (2005). Quality standards, conventions and the governance of global value chains, 34(1). doi:. Economy and Society, 34(1), 1-31. doi:10.1080/0308514042000329315

Tallontire, A., Dixon, J., y Benton, T. (2012). A Review of the Literature and Knowledge of Standards an Certification Systems in Agricultural Production and Farming Systems. NRI Working Paper Series on Sustainability Standard. 